\title{
Etiological studies of traumatic injury: are we measuring the right outcome?
}

\author{
William Pickett, John R Hoey
}

\begin{abstract}
A description of the issue
Many etiological studies investigate risk factors for the occurrence of acute injury events. The epidemiological approaches used in such investigations include case-control studies, where people are classified based on the occurrence or non-occurrence of an injury and the exposure experience of the two groups is compared. Alternatively, some studies use a cohort approach, which in its simplest form, compares the injury experiences of people over time who have and have not been exposed to a potential risk factor. We have been considering the methods currently used to classify injury outcomes in these types of studies and would like to share these ideas with the readers of Injury Prevention, in the hopes of involving some thought and debate.
\end{abstract}

By definition, the purpose of etiological studies is to quantitatively assess the strength of potential risk factors for injury. For example, an investigation might examine how family income levels (or some other measure of socioeconomic status) are associated with the occurrence of certain childhood injuries. What we would normally do in this situation is to classify the outcomes in a dichotomous fashion (that is, as injury or no injury), and perform our analysis based upon this outcome measure. While this is a straightforward and generally accepted method, we wonder whether it is appropriate for all etiological studies. Many injury control specialists view injuries as a form of disease, and this view provides us with a conceptual framework from which preventive interventions can be formulated. But what if acute injury events are really manifestations of a disease process? What if that process is real and the injury only a proxy measurement for the existence of this disease?

If we were to accept this idea it might lead to a change in the way we view and measure injuries. Instead of considering injuries to be acute events leading to physical harm, we would need to broaden the definition to include a disease where individuals consistently (and perhaps systematically) place themselves at risk for injury.

What would this injury 'disease' look like? It is difficult to debate whether this disease actually exists in the absence of a clear, working definition. Although we have not developed this definition in any detail, the disease might be characterized, in broad terms, as including a personal, social (and possibly even, genetic) environment that fosters or accepts risk taking on a day-to-day basis, in combination with a reduced ability to avoid episodes of risk. This idea is different from the old theory of 'accident proneness'. This definition should be based upon exposures that foster risk and a lack of risk avoidance, as opposed to the fatalistic view that some people have an innate susceptibility to injuries for no apparent reason.

To illustrate, one of our research group's major interests is farm injuries. A subpopulation consistently at risk in this setting are young boys. The personal and social characteristics of some farm environments, that foster risk and lead to its acceptance, include the constant need for manual labour combined with the expectation that adolescent males are capable of handling hazardous tasks and long working hours. Coupled with these environmental risks is the fact that young boys are still maturing and may lack the stamina and coordination to consistently avoid farm work hazards. All these factors create a situation where the boys are consistently working at high risk. This provides one example of a set of environmental and personal conditions that might constitute this 'disease state' so that injuries occur frequently.

\section{Implications of this idea for etiological studies of injury}

There are some important methodological implications associated with this idea. As injury control researchers, it might be important to refocus some of our etiological work on associations between potential risk factors and the presence of this disease, and not continue to focus solely on risk factors for injuries. For example, instead of examining only associations between socioeconomic status and the occurrence of injuries, we should also investigate the association between socioeconomic status and the occurrence of the risk taking disease. This could provide a clearer picture of the true etiology of the conditions that lead to injury.

Our current method of classifying diseased and non-diseased people in injury studies (that is injured/non-injured) might obscure the true etiological relationships of importance. Those who have not yet had an injury would currently be misclassified as 'non-diseased'. The classification of those who are truly nondiseased would be unaffected. If a true association existed between some factor and the occur- 
rence of this newly defined disease, it would be difficult to detect if outcomes were determined only according to the presence or absence of injury events, as opposed to this more refined measure.

Further, in the development of multivariable etiological models, one guiding principle is never to adjust for variables unless we understand how they relate to both the exposure and the outcome. If one accepts our proposition (that we are not measuring this outcome appropriately), this means that we may not be developing appropriate etiological models. We might be considering variables as risk factors contributing to the occurrence of disease when, in fact, they are actually part of the disease process. Adjustments for these variables could lead to biased estimates of risk being attributed to the underlying risk factors. More importantly, this would lead to inappropriate recommendations for preventive intervention.

\section{Conclusion}

The purpose of this Opinion is to share thoughts about whether injury researchers are employing the correct outcome in the conduct of etiological studies. We offer these ideas not as definitive statements based on objective information, but as possibilities that require consideration and debate. If the ideas are legitimate they are certain to have some application in future investigations.

\title{
DISSENT
}

\section{Risk taking disease}

\author{
Stephen Jarvis
}

The Opinion paper by Pickett and Hoey opens a can of worms! Any dissent from these slippery worms may prove difficult, but I hope to convince readers that this particular can should be kept shut - but then reopened from the other end!

The reasoning will follow the line - what is this new disease (risk taking disease)? - how would I know I had it? - can this disease actually be located in a specific person? - how would an epidemiologist find its associated risk factors? - how does risk taking disease stand up to accepted definitions of disease? - what alternative might improve the situation?

\section{What is risk taking disease?}

Although the authors admit that they have not 'developed this definition in any detail' it is possible to tease out a couple of summary strands describing their concept. For instance, they suggest that 'a record of an injury' may be 'only a proxy measurement for the existence of this disease'. Later they suggest 'the definition [of this disease] should be based upon exposures that foster risk and a lack of risk avoidance'.

Community Child Health, Donald Court House, University of Newcastle upon Tyne, 13 Walker Terrace, Gateshead, NE8 1EB, UK

Correspondence to: Professor Jarvis.

\section{How would I know when I've got risk} taking disease

First of all, I don't seem to need an injury. Rather it seems clear that the authors would characterise this disease by measures of 'exposures that foster risk' and separate measures that capture 'ability to avoid episodes of risks'. Perhaps this might be encapsulated as 'what hazards does the environment present and how do people react to this?' To address the second half of this question one might need to go beyond behaviour variations in the face of specified hazards to include attitudes towards risk seeking, or even further to include physical and psychosocial variations in risk perception. Whatever the refinements, however, a central dimension is the measurement of exposure to risk of injury, irrespective of whether the balance of attributability is environmental or behavioral.

How would an epidemiologist study risk taking disease?

Comparing the rate of events per unit (or degree of) exposure to postulated risk factors is a basic epidemiological tool when examining aetiology. Defining disease by exposure to risk factors is, therefore, likely to be circular. If risk taking disease is defined as above, then the relevant risk factor/exposures are those that might predict this particular combination of primary exposures (that is, risk taking disease). There is a danger that rates of this disease per unit of secondary risk factor exposure will fail to predict ultimate injury outcomes. For instance, injury and secondary exposure could be independently related to its frequency (that is the disease may be a confounder). Furthermore, in experimental studies, the disease might be 'cured' by altering the physical environment in which somebody lives! 